\section{WHAT IS NOT "EFFECTIVE" IN MILD TO MODERATE DEPRESSION: ANTIDEPRESSANTS OR THE HAMILTON RATING SCALE FOR DEPRESSION?}

\section{To the Editor:}

Recent evidence in the literature ${ }^{1}$ raised the point that the effect of antidepressants for mild and moderate depression may be minimal or non-existent. This is a very important question that leads us to discuss the level of evidence that supports the widespread use of antidepressants for less severe forms of depression. In counterpart, the burden of depression is also well known and antidepressants are the most common treatment approach available.

Although evidence favoring the lack of efficacy of antidepressants seems to be strong, one could argue that maybe these results can be attributed to psychometric pitfalls in the proprieties of the instruments used to define response in randomized clinical trials, such as the Hamilton Rating Scale for Depression-17 items (HAM-D $\left.{ }_{17}\right)^{2}$ This extremely simple problem could have major implications in clinical practice over the world.

The HAM- $D_{17}$ has being criticized for several flaws, including lack of uni-dimentionallity, demonstrated by both classical $\left.\right|^{3,4}$ and modern psychometric methods ${ }^{3}$ such as Rasch Analysis and Item Response Analysis. Santor and colleagues, ${ }^{5}$ analyzing a community sample of depressed patients, could show specifically that some items of $H A M-D_{17}$ were not effective at discriminating different degrees of depressive severity, particularly within mild and moderate levels. If a scale is composed mostly of items measuring moderate to very severe depression, the measurement scale would have great difficulty in discriminating mild and moderate cases as both would be characterized by low scores on all items. It is like an "industrial thermometer being used to measure a temperature of a baby."

In addition, one should also note that although $\mathrm{HAM}-\mathrm{D}_{17}$ have a maximum score of 52, mild to moderate depressions have, by definition, scores with $<13$ and 18 points, respectively. Moreover, in almost all randomized clinical trials of antidepressants for depressive disorders there is a classical placebo response of $20 \%$ to $40 \%$ and very few patients achieve full remission after treatment completion, reducing considerably the margin to show the true effect of antidepressants over placebo in such a small number of points in the measurement scale for these severity levels.

We do agree that prescribers, policy makers, and consumers must be aware that the efficacy of antidepressants for depression largely has been established on the basis of studies that have included mostly more severe forms of depression. However, we are hypothesizing that an alternative explanation for the lack of efficacy in mild to moderate cases could be attributable to psychometric pitfalls in HAM-D ${ }_{17} \cdot 3,5$ Moreover, the same hypothesis could be a reasonable explanation for discrete effectiveness reported for psychotherapies in major depression after the adjustment for publication bias. ${ }^{6}$

In conclusion, the utility of HAM- $D_{17}$ as a gold standard measure for comparing treatment effects of mild to moderate depression still needs to be established. Without that we could condemn the "wrong suspect" (antidepressants) letting free the "real guilty" (HAM-D ${ }_{17}$ ).
Sincerely, Giovanni Abrahão Salum, MD, PhD Student

Gisele Gus Manfro, MD, PhD

Marcelo P. Fleck, MD, PhD

\section{REFERENCES}

1. Fournier JC, DeRubeis RJ, Hollon SD, et al. Antidepressant drug effects and depression severity: a patient-level meta-analysis. JAMA. 2010;303(1):47-53.

2. Hamilton M. A rating scale for depression. J Neurol Neurosurg Psychiatry. 1960;23:56-62.

3. Bagby RM, Ryder AG, Schuller DR, Marshall MB. The Hamilton Depression Rating Scale: has the gold standard become a lead weight? Am J Psychiatry. 2004;161(12):2163-2177.

4. Fleck MP, Chaves ML, Poirier-Littre MF, et al. Depression in France and Brazil: factorial structure of the 17-item Hamilton Depression Scale in inpatients. J Nerv Ment Dis. 2004:192(2):103-110.

5. Santor DA, Coyne JC. Examining symptom expression as a function of symptom severity: item performance on the Hamilton Rating Scale for Depression. Psychol Assess. 2001;13(1):127-139.

6. Cuijpers P, Smit F, Bohlmeijer E, Hollon SD, Andersson G. Efficacy of cognitive-behavioural therapy and other psychological treatments for adult depression: metaanalytic study of publication bias. $\mathrm{Br} J$ Psychiatry. 2010;196:173-178.

Dr. Salum is a PhD student, Dr. Manfro is associate professor, and Dr. Fleck associate professor, all at the Mood and Anxiety Disorders Program of Hospital de Clínicas de Porto Alegre Post-Graduate Prog Psychiatry Universidade Federal do Rio Grande do Sul, Brazil.

Faculty Disclosures: Dr. Fleck and Dr. Manfro receive a senior researcher CNPq scholarship. Dr Salum receives a CAPES doctoral scholarship.

Submitted for publication: March 1, 2010; Accepted for publication: June 4, 2010. First published online: April 1, 2011

Please direct all correspondence to: Giovann Abrahao Salum, MD, Ramiro Barcelos, 2350 (room 2202) / 90035-003; E-mail: gsalumir@gmail.com 